\title{
Longitudinal flow and onset of deconfinement
}

\author{
Hannah Petersen * \\ Institut für Theoretische Physik, Johann Wolfgang Goethe-Universität, Max-von-Laue-Str. 1, \\ D-60438 Frankfurt am Main, Germany \\ E-mail: petersen@th.physik.uni-frankfurt.de
}

\section{Marcus Bleicher ${ }^{\dagger}$}

Institut für Theoretische Physik, Johann Wolfgang Goethe-Universität, Max-von-Laue-Str. 1, D-60438 Frankfurt am Main, Germany

E-mail: bleicherdth.physik.uni-frankfurt.de

\begin{abstract}
The effects of the onset of deconfinement on longitudinal and transverse flow are studied. First, we analyze longitudinal pion spectra from $E_{\text {lab }}=2 A \mathrm{GeV}$ to $\sqrt{s_{\mathrm{NN}}}=200 \mathrm{GeV}$ within Landau's hydrodynamical model and the UrQMD transport approach. From the measured data on the widths of the pion rapidity spectra, we extract the sound velocity $c_{s}^{2}$ in the early stage of the reactions. It is found that the sound velocity has a local minimum (indicating a softest point in the equation of state, EoS) at $E_{\text {beam }}=30 \mathrm{~A} \mathrm{GeV}$. This softening of the EoS is compatible with the assumption of the formation of a mixed phase at the onset of deconfinement. Furthermore, the energy excitation function of elliptic flow $\left(v_{2}\right)$ from $E_{\text {beam }}=90 A \mathrm{MeV}$ to $\sqrt{s_{\mathrm{NN}}}=200 \mathrm{GeV}$ is explored within the UrQMD framework and discussed in the context of the available data. The transverse flow should also be sensitive to changes in the equation of state. Therefore, the underestimation of elliptic flow by the UrQMD model calculation above $E_{\text {lab }}=30 \mathrm{~A} \mathrm{GeV} \mathrm{might}$ also be explained by assuming a phase transition from a hadron gas to the quark gluon plasma around this energy. This would be consistent with the model calculations, indicating a transition from hadronic matter to "string matter" in this energy range.
\end{abstract}

Critical Point and Onset of Deconfinement

July 3-6 2006

Florence, Italy

${ }^{*}$ H.P. gratefully acknowledges fi nancial support by a stipendship of the Deutsche Telekom-Stiftung † Speaker. 


\section{Introduction}

Over the last years, a wealth of detailed data in the $20 \mathrm{~A}-160 \mathrm{~A} \mathrm{GeV}$ energy regime has become available. The systematic study of these data revealed surprising (non-monotonous) structures in various observables around $30 \mathrm{~A} \mathrm{GeV}$ beam energy. Most notable irregular structures in that energy regime include,

- the sharp maximum in the $\mathrm{K}^{+} / \pi^{+}$ratio [1,2],

- a step in the transverse momentum excitation function (as seen through $\left\langle m_{\perp}\right\rangle-m_{0}$ ) $[2,3]$,

- an apparent change in the pion per participant ratio [2] and

- increased ratio fluctuations (due to missing data at low energies it is unknown if this is a local maximum or an ongoing increase of the fluctuations) [4].

It has been speculated, that these observation hint towards the onset of deconfi nement already at $30 \mathrm{~A} \mathrm{GeV}$ beam energy. Indeed, increased strangeness production [5] and enhanced fluctuations have long been predicted as a sign of QGP formation $[6,7,8,9,10,11,12]$ within different frameworks and observables. The suggestion of an enhanced strangeness to entropy ratio $(\sim K / \pi)$ as indicator for the onset of QGP formation was especially advocated in [13]. Also the high and approximately constant $K^{ \pm}$inverse slopes of the $m_{T}$ spectra above $\sim 30 \mathrm{~A} \mathrm{GeV} \mathrm{-} \mathrm{the} \mathrm{'step'} \mathrm{-} \mathrm{was}$ also found to be consistent with the assumption of a parton $\leftrightarrow$ hadron phase transition at low SPS energies [14, 15]. Surprisingly, transport simulations (supplemented by recent lattice QCD (IQCD) calculations) have also suggested that partonic degrees of freedom might already lead to visible effects at $\sim 30 \mathrm{~A} \mathrm{GeV}[16,17,18]$. Finally, the comparison of the thermodynamic parameters $T$ and $\mu_{B}$ extracted from the transport models in the central overlap region [19] with the experimental systematics on chemical freeze-out confi gurations $[20,21,22]$ in the $T-\mu_{B}$ plane do also suggest that a fi rst glimpse on a deconfi ned state might be possible around $10 \mathrm{~A}-30 \mathrm{~A} \mathrm{GeV}$.

In the first part of this paper, we explore wether similar irregularities are also present in the excitation function of longitudinal observables, namely rapidity distributions. Here we employ Landau's hydrodynamical model and the UrQMD transport approach. In the second part, we focus on the excitation function of transverse fbw $\left(v_{2}\right)$ and discuss UrQMD results in the context of the available data.

\section{Longitudinal flow}

It became popular to interpret relativistic heavy ion reactions with Landau's hydrodynamical model [23, 24, 25, 26, 27, 28] (for recent applications of this model to relativistic nucleus-nucleus interactions see $[29,30,31,32,33,34])$. Therefore we will use this simple hydrodynamical picture as a baseline for the model and data comparison. The main physics assumptions of Landau's picture are: The collision of two Lorentz-contracted hadrons or nuclei leads to full thermalization in a volume of size $V m_{p} / \sqrt{s}$. This justifi es the use of thermodynamics and establishes the system size and energy dependence. Usually a simple equation of state $p=\varepsilon / 3$ is assumed. Chemical potentials are usually assumed to vanish. The main results derived from these assumptions are: A 

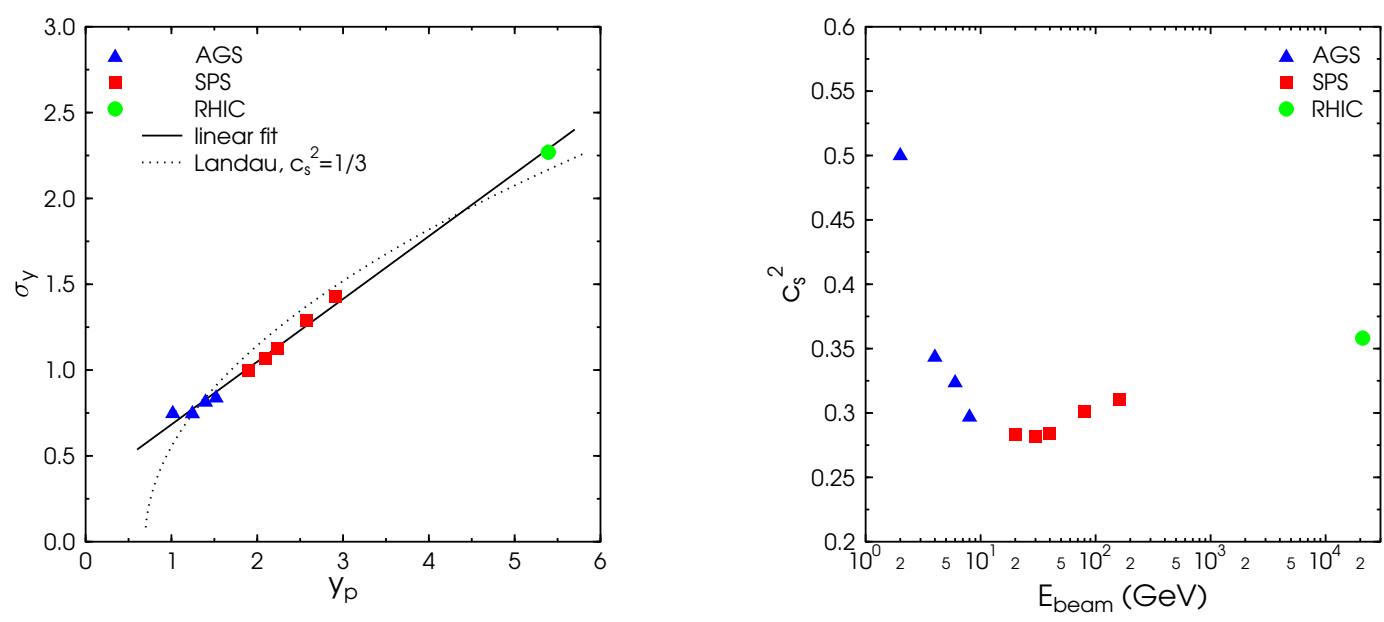

Figure 1: Left: The root mean square $\sigma_{y}$ of the rapidity distributions of negatively charged pions in central $\mathrm{Pb}+\mathrm{Pb}(\mathrm{Au}+\mathrm{Au})$ reactions as a function of the beam rapidity $y_{p}$. The dotted line indicates the Landau model prediction with $c_{s}^{2}=1 / 3$, while the full line shows a linear fit through the data points. Data (full symbols) are taken from $[3,32,36,37]$. The statistical errors given by the experiments are smaller than the symbol sizes. Systematic errors are not available. Right: Speed of sound as a function of beam energy for central $\mathrm{Pb}+\mathrm{Pb}(\mathrm{Au}+\mathrm{Au})$ reactions as extracted from the data using Eq. (2.3). The statistical errors (not shown) are smaller than $3 \%$.

universal formula for the produced entropy, determined mainly by the initial Lorentz contraction and Gaussian rapidity distributions, at least for newly produced particles. Under the condition that $c_{s}$ is independent of temperature, the rapidity density is given by [27, 35]:

$$
\frac{d N}{d y}=\frac{K s_{\mathrm{NN}}^{1 / 4}}{\sqrt{2 \pi \sigma_{y}^{2}}} \exp \left(-\frac{y^{2}}{2 \sigma_{y}^{2}}\right)
$$

with

$$
\sigma_{y}^{2}=\frac{8}{3} \frac{c_{s}^{2}}{1-c_{s}^{4}} \ln \left(\sqrt{s_{\mathrm{NN}}} / 2 m_{p}\right)
$$

where $K$ is a normalisation factor and $m_{p}$ is the proton mass.

Let us now analyze the available experimental data on rapidity distributions of negatively charged pions in terms of the Landau model. Fig. 1 (left) shows the measured root mean square $\sigma_{y}$ of the rapidity distribution of negatively charged pions in central $\mathrm{Pb}+\mathrm{Pb}(\mathrm{Au}+\mathrm{Au})$ reactions as a function of the beam rapidity. The dotted line indicates the Landau model predictions with the commonly used constant sound velocity $c_{s}^{2}=1 / 3$. The full line shows a linear fit through the data points, while the data points $[3,32,36,37]$ are depicted by full symbols.

At a fi rst glance the energy dependence looks structureless. The data seem to follow a linear dependence on the beam rapidity $y_{p}$ without any irregularities. However, the general trend of the rapidity widths is also well reproduced by Landau's model with an equation of state with a fi xed speed of sound. Nevertheless, there seem to be systematic deviations. At low AGS energies and at RHIC, the experimental points are generally underpredicted by Eq. (2.2), while in the SPS 


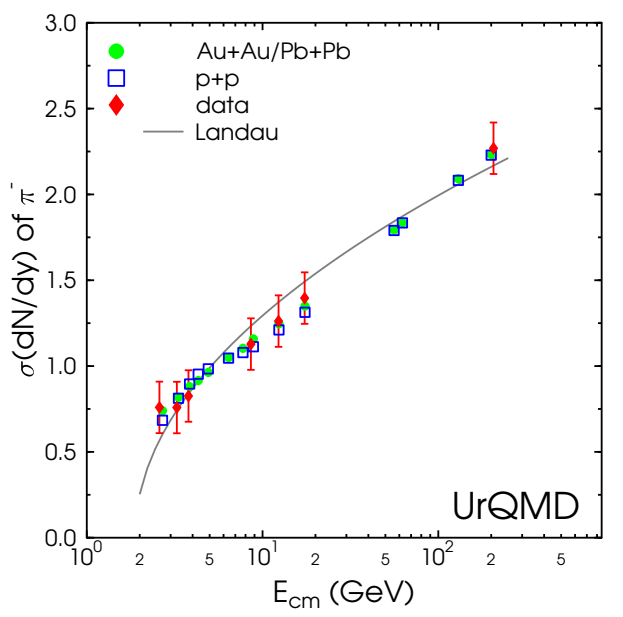

Figure 2: The root mean square of the rapidity distribution of negatively charged Pions in central $\mathrm{Au}+\mathrm{Au} / \mathrm{Pb}+\mathrm{Pb}$ and Proton+Proton reactions as a function of the center of mass energy. UrQMD calculations for $\mathrm{Au}+\mathrm{Au} / \mathrm{Pb}+\mathrm{Pb}$ are denoted by full circles, the pp results are shown by open squares. The prediction from Landau's model is given by the line (Eq. 2.1). Data [32] are depicted by full diamonds.

energy regime Landau's model overpredicts the widths of the rapidity distributions. Exactly these deviations from the simple Landau picture do allow to gain information on the equation of state of the matter produced in the early stage of the reaction. By inverting Eq. (2.2) we can express the speed of sound $c_{s}^{2}$ in the medium as a function of the measured width of the rapidity distribution:

$$
c_{s}^{2}=-\frac{4}{3} \frac{\ln \left(\sqrt{s_{\mathrm{NN}}} / 2 m_{p}\right)}{\sigma_{y}^{2}}+\sqrt{\left[\frac{4}{3} \frac{\ln \left(\sqrt{s_{\mathrm{NN}}} / 2 m_{p}\right)}{\sigma_{y}^{2}}\right]^{2}+1} .
$$

Let us now investigate the energy dependence of the sound velocities extracted from the data. Fig. 1 (right) shows the speed of sound as a function of beam energy for central $\mathrm{Pb}+\mathrm{Pb}(\mathrm{Au}+\mathrm{Au})$ reactions as obtained from the data using Eq. (2.3). The sound velocities exhibit a clear minimum (usually called the softest point) around a beam energy of $30 \mathrm{~A} \mathrm{GeV}$. A localized softening of the equation of state is a long predicted signal for the mixed phase at the transition energy from hadronic to partonic matter $[38,39,40]$.

To test this hypothesis the same observable has also been calculated using the UrQMD model (v2.2) [41, 42]. This transport model takes into account the formation and multiple rescattering of hadrons and dynamically describes the generation of pressure in the hadronic expansion phase. It involves also interactions of (di-)quarks, however gluonic degrees of freedom are not treated explicitly, but are implicitly present in strings. This simplifi ed treatment is generally accepted to describe Proton-Proton and Proton-nucleus interactions.

As depicted in Fig. 2 the UrQMD predictions (full circles) for the rapidity widths of negatively charged pions in $\mathrm{Au}+\mathrm{Au}(\mathrm{Pb}+\mathrm{Pb})$ reactions are in line with the experimental data [32] (full diamonds) and Landau's hydrodynamical model (full line). A rather surprising observation is that the calculated rapidity widths of $\pi^{-}$in pp interactions (open squares) are identical to the AA results. Together with the previous discussion, it seems that the equation of state in the transport model is also soft in the SPS regime. Thus, the nature of the softest point remains unclear. 

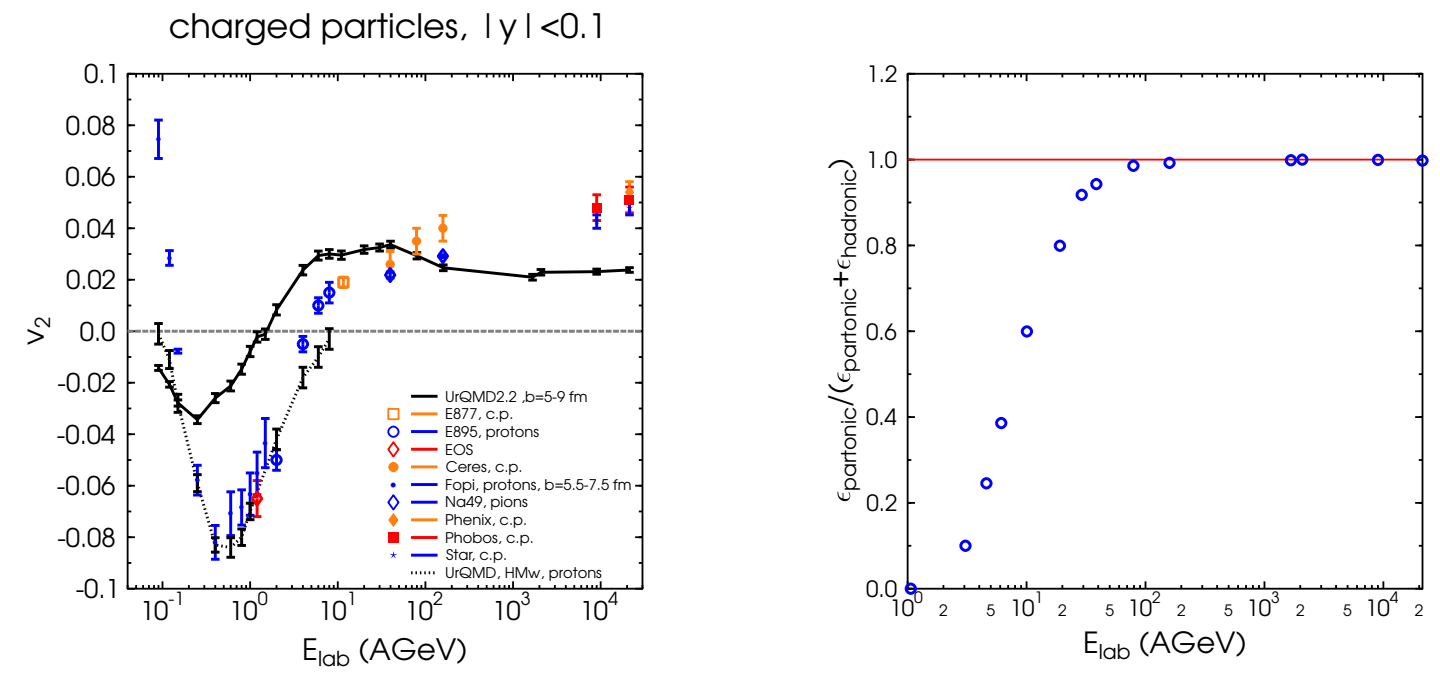

Figure 3: Left: The calculated energy excitation function of elliptic flow of charged particles in $\mathrm{Au}+\mathrm{Au} / \mathrm{Pb}+\mathrm{Pb}$ collisions in mid-central collisions ( $\mathrm{b}=5-9 \mathrm{fm}$ ) with $|y|<0.1$ (black line). This curve is compared to data from different experiments for mid-central collisions. For E895 [46, 47] and FOPI [48] there is the elliptic flow of protons and for NA49 [49] it is the elliptic flow of pions. For E877, CERES [50, 51, 52], PHENIX [53], PHOBOS [54] and STAR [55] there is data for the charged particle flow. The dotted line in the low energy regime depict UrQMD calculations with the mean field [60]. Right: Calculated fraction of energy density in unformed hadrons with $|y|<0.5$ and in a cylindrical volume with transverse radius $r=3$ $\mathrm{fm}$ and length $h=3 / \gamma_{C M} \mathrm{fm}$ as a function of the beam energy for central $\mathrm{Pb}+\mathrm{Pb}(\mathrm{Au}+\mathrm{Au}$ ) reactions.

\section{Transverse flow}

Let us now look at the dynamics of the system perpendicular to the beam direction. The transverse fbw is intimately connected to the pressure gradients. Therefore, it is sensitive to the equation of state (EoS) and might be used to search for abnormal matter states and phase transitions $[43,44,45]$. Especially the second coeffi cient of the Fourier expansion of the azimuthal distribution of the emitted particles $\left(v_{2}\right)$ is a valuable tool to gain insight into the expanding stage of the fir reball.

The excitation function of charged particle elliptic fbw is compared to data over a wide energy range (Fig. 3 (left)), i.e from $E_{\text {beam }}=90 \mathrm{~A} \mathrm{MeV}$ to $\sqrt{s_{N N}}=200 \mathrm{GeV}$. The squeeze-out effect at low energies and the change to in-plane emission at higher energies is nicely observed in the excitation function. The symbols indicate the data for charged particles from different experiments. Note however, that in the low energy regime there are only experimental data points for protons. For beam energies below $2 \mathrm{~A} \mathrm{GeV}$ most of the charged particles are also protons because there is not enough energy to produce many new particles. Going to higher energies the elliptic fbw of pions and charged particles are very similar. The rapidity cut of $|y|<0.1$ has been used for the whole energy range despite the fact that the data at higher energies is within $|y|<0.5$. This has been done to avoid too much changes in the parameters and this choice gives reasonable results over the whole energy range. We have checked that the results at higher energies are not affected by the choice of this narrower rapidity window.

At low energies $E_{\text {beam }} \sim 0.1-6 \mathrm{~A} \mathrm{GeV}$ the squeeze-out effect, i.e. the elliptic fbw out-of- 
plane, is clearly seen in the data as well as in the calculations, especially when the mean fi eld is considered. At such energies, it is well known that both the mean fi eld and the two-body collision are equally important to reproduce quantitatively the experimental results $[56,57,58]$. In this paper we adopt a hard equation of state with momentum dependence (HMw) which was updated recently in the UrQMD model $[59,60]$.

In the SPS regime the model calculations are quite in line with the data, especially with the NA49 results. For a more detailed discussion of directed and elliptic fbw results from UrQMD-2.2 the reader is referred to $[61,62,63]$. Above $E_{\text {lab }}=160 \mathrm{~A} \mathrm{GeV}$ the calculation underestimates the elliptic fbw. At the highest RHIC energy there are about $5 \% \mathrm{fbw}$ in the data while the model calculation provides only half of this value. This can be explained by assuming a lack of pressure in the transport model at these energies.

It is possible that above the energy range about $E_{\text {lab }}=30 \mathrm{AGeV}$ partonic interactions have to be taken into account to describe the data as suggested in $[18,64,65]$. How can we analyse this question in the model, since there are no partonic degrees of freedom explicitly incorporated? In the current model exists a formation time for hadrons produced in the string fragmentation. The leading hadrons of the fragmenting strings contain the valence quarks of the original excited hadron. These (di-)quark string ends are allowed to interact during their formation time with a reduced cross section defi ned by the additive quark model. Other "pre-hadrons" from the fragmenting string are not allowed to interact before the coalescence of the produced quarks. Thus, because the unformed hadrons do not interact with others during their formation time, the effective pressure is reduced and only build up from the density of the formed hadrons.

To illuminate this, we have calculated the energy density during heavy ion collisions at different beam energies. From this, we extract the time corresponding to the maximum value of the total energy density. Fig. 3 (right) shows the fraction of the energy density that is deposited in the "unformed hadrons" $\left(\varepsilon_{\text {partonic }} /\left(\varepsilon_{\text {partonic }}+\varepsilon_{\text {hadronic }}\right)\right)$. I.e. all string fragments within their formation time are dubbed as "partonic". The fraction of $\varepsilon_{\text {partonic }}$ starts at zero for low energies and then rises fast to almost $100 \%$. Note that this fraction reaches $90 \%$ already around $30 \mathrm{AGeV}$ beam energy, similar to the energy region where a phase transition is expected. As one can see, the energy density of the formed hadrons ( $\varepsilon_{\text {hadronic }}$ ) is much smaller than the total value, therefore the effective pressure of the formed hadrons alone in the model seems to be too small to generate enough $v_{2}$. Thus, this fi nding supports the interpretation of the need for initial pressure from non-hadronic matter already at low SPS energies.

\section{Conclusion}

In conclusion, we have explored the excitation functions of the rapidity widths and of elliptic $\mathrm{fbw}$ of (negatively) charged pions in $\mathrm{Pb}+\mathrm{Pb}(\mathrm{Au}+\mathrm{Au})$ collisions. The following observations can be made:

- The rapidity spectra of pions produced in central nucleus-nucleus reactions at all investigated energies can be well described by single Gaussians.

- The energy dependence of the width of the pion rapidity distribution follows the prediction of Landau's hydrodynamical model if a variation of the sound velocity is taken into account. 
- The speed of sound excitation function extracted from the data has a pronounced minimum (softest point) at $E_{\text {beam }}=30 \mathrm{~A} \mathrm{GeV}$.

- The UrQMD model describes the rapidity widths data well, but underestimates the elliptic fbw from the higher SPS energy on.

- The softest point coincides with the rapid rise of "partonic" degrees of freedom in the present model.

- This softest point might be due to the formation of a mixed phase indicating the onset of deconfi nement at this energy.

Further explorations of this energy domain is needed and can be done at the future FAIR facility and by CERN-SPS and BNL-RHIC experiments.

\section{Acknowledgements}

This work was supported by GSI and BMBF. This work used computational resources provided by the Center for Scientifi c Computing at Frankfurt (CSC).

\section{References}

[1] S. V. Afanasiev et al. [The NA49 Collaboration], Phys. Rev. C 66 (2002) 054902 [arXiv:nucl-ex/0205002].

[2] M. Gazdzicki et al. [NA49 Collaboration], J. Phys. G 30 (2004) S701 [arXiv:nucl-ex/0403023].

[3] C. Blume, J. Phys. G: Nucl. Part. Phys. 31, S57 (2005)

[4] C. Roland [NA49 Collaboration], J. Phys. G 31 (2005) S1075.

[5] P. Koch, B. Muller and J. Rafelski, Phys. Rept. 142 (1986) 167.

[6] M. Bleicher, S. Jeon and V. Koch, Phys. Rev. C 62 (2000) 061902 [arXiv:hep-ph/0006201].

[7] S. Jeon, L. Shi and M. Bleicher, Phys. Rev. C 73, 014905 (2006) [arXiv:nucl-th/0506025].

[8] E. V. Shuryak and M. A. Stephanov, Phys. Rev. C 63 (2001) 064903 [arXiv:hep-ph/0010100].

[9] H. Heiselberg and A. D. Jackson, Phys. Rev. C 63 (2001) 064904 [arXiv:nucl-th/0006021].

[10] B. Muller, Nucl. Phys. A 702 (2002) 281 [arXiv:nucl-th/0111008].

[11] M. Gazdzicki, M. I. Gorenstein and S. Mrowczynski, Phys. Lett. B 585, 115 (2004) [arXiv:hep-ph/0304052].

[12] M. I. Gorenstein, M. Gazdzicki and O. S. Zozulya, Phys. Lett. B 585, 237 (2004) [arXiv:hep-ph/0309142].

[13] M. Gazdzicki and M. I. Gorenstein, Acta Phys. Polon. B 30, 2705 (1999).

[14] M. I. Gorenstein, M. Gazdzicki and K. A. Bugaev, Phys. Lett. B 567, 175 (2003) [arXiv:hep-ph/0303041].

[15] Y. Hama, F. Grassi, O. Socolowski, T. Kodama, M. Gazdzicki and M. Gorenstein, Acta Phys. Polon. B 35 (2004) 179. 
[16] H. Weber, C. Ernst, M. Bleicher et al., Phys. Lett. B 442, 443 (1998).

[17] E. L. Bratkovskaya et al., Phys. Rev. Lett. 92, 032302 (2004)

[18] E. L. Bratkovskaya et al., Phys. Rev. C 69, 054907 (2004)

[19] L. V. Bravina et al., Phys. Rev. C 60, 024904 (1999), Nucl. Phys. A 698, 383 (2002).

[20] P. Braun-Munzinger and J. Stachel, Nucl. Phys. A 606, 320 (1996).

[21] P. Braun-Munzinger and J. Stachel, Nucl. Phys. A 638, 3 (1998).

[22] J. Cleymans and K. Redlich, Phys. Rev. C 60, 054908 (1999).

[23] E. Fermi, Prog. Theor. Phys. 5, 570 (1950).

[24] L. D. Landau, Izv. Akad. Nauk Ser. Fiz. 17, 51 (1953).

[25] S. Z. Belenkij and L. D. Landau, Usp. Fiz. Nauk 56, 309 (1955).

[26] P. Carruthers and M. Doung-van, Phys. Rev. D 8, 859 (1973).

[27] P. Carruthers, Annals N.Y.Acad.Sci. 229, 91 (1974).

[28] P. A. Carruthers, LA-UR-81-2221

[29] J. Stachel and P. Braun-Munzinger, Phys. Lett. B 216, 1 (1989).

[30] P. Steinberg, arXiv:nucl-ex/0405022.

[31] M. Murray, arXiv:nucl-ex/0404007.

[32] G. Roland, Talk presented at Quark Matter 2004, see proceedings.

[33] M. Bleicher, arXiv:hep-ph/0504207.

[34] M. Bleicher, arXiv:hep-ph/0509314.

[35] E. V. Shuryak, Yad. Fiz. 16, 395 (1972).

[36] J.Klay et al. [E895 Collaboration], Phys. Rev. C 68, 054905 (2003)

[37] I.G. Bearden et al. [Brahms Collaboration], Phys. Rev. Lett. 94, 162301 (2005)

[38] C. M. Hung and E. V. Shuryak, Phys. Rev. Lett. 75, 4003 (1995) [arXiv:hep-ph/9412360].

[39] D. H. Rischke, Y. Pursun, J. A. Maruhn, H. Stoecker and W. Greiner, Heavy Ion Phys. 1, 309 (1995) [arXiv:nucl-th/9505014].

[40] J. Brachmann, A. Dumitru, H. Stoecker and W. Greiner, Eur. Phys. J. A 8, 549 (2000) [arXiv:nucl-th/9912014].

[41] S.A. Bass et al., Prog. Part. Nucl. Phys. 42, 255 (1998).

[42] M. Bleicher et al., J. Phys. G 25, 1859 (1999).

[43] H. Stöcker, J. A. Maruhn and W. Greiner, Z. Phys. A 290 (1979) 297.

[44] J. Hofmann, H. Stöcker, U. W. Heinz, W. Scheid and W. Greiner, Phys. Rev. Lett. 36 (1976) 88.

[45] H. Stöcker and W. Greiner, Phys. Rept. 137 (1986) 277.

[46] C. Pinkenburg et al. [E895 Collaboration], Prepared for Centennial Celebration and Meeting of the American Physical Society (Combining Annual APS General Meeting and the Joint Meeting of the APS and the AAPT), Atlanta, Georgia, 20-26 Mar 1999 
[47] P. Chung et al. [E895 Collaboration], Phys. Rev. C 66 (2002) 021901 [arXiv:nucl-ex/0112002].

[48] A. Andronic et al. [FOPI Collaboration], Phys. Lett. B 612 (2005) 173 [arXiv:nucl-ex/0411024].

[49] C. Alt et al. [NA49 Collaboration], Phys. Rev. C 68 (2003) 034903 [arXiv:nucl-ex/0303001].

[50] K. Filimonov et al. [CERES/NA45 Collaboration], arXiv:nucl-ex/0109017.

[51] J. Slivova [CERES/NA45 Collaboration], Nucl. Phys. A 715 (2003) 615 [arXiv:nucl-ex/0212013].

[52] S.I.Esumi, J. Slivova, J. Milosevic for CERES Collaboration SFIN, year XV, Series A: Conferences, No. A2(2002)

[53] S. Esumi [PHENIX Collaboration], Nucl. Phys. A 715 (2003) 599 [arXiv:nucl-ex/0210012].

[54] S. Manly et al. [PHOBOS Collaboration], Nucl. Phys. A 715 (2003) 611 [arXiv:nucl-ex/0210036].

[55] R. L. Ray [STAR Collaboration], Nucl. Phys. A 715 (2003) 45 [arXiv:nucl-ex/0211030].

[56] P. Danielewicz, Nucl. Phys. A 661 (1999) 82 [arXiv:nucl-th/9907098].

[57] P. Danielewicz, R. A. Lacey, P. B. Gossiaux, C. Pinkenburg, P. Chung, J. M. Alexander and R. L. McGrath, Phys. Rev. Lett. 81 (1998) 2438 [arXiv:nucl-th/9803047].

[58] Q. b. Pan and P. Danielewicz, Phys. Rev. Lett. 70 (1993) 2062 [Erratum-ibid. 70 (1993) 3523].

[59] Q. Li, Z. Li, S. Soff, M. Bleicher and H. Stöcker, J. Phys. G: Nucl. Part. Phys. 32 (2006) 151

[60] Q. f. Li, Z. x. Li, S. Soff, M. Bleicher and H. Stöcker, J. Phys. G 32 (2006) 407 [arXiv:nucl-th/0601047].

[61] H. Petersen, Q. Li, X. Zhu and M. Bleicher, Phys. Rev. C in print [arXiv:hep-ph/0608189].

[62] X. 1. Zhu, M. Bleicher and H. Stoecker, J. Phys. G 32 (2006) 2181 [arXiv:nucl-th/0601049].

[63] X. 1. Zhu, M. Bleicher and H. Stoecker, Phys. Rev. C 72, 064911 (2005) [arXiv:nucl-th/0509081].

[64] H. Weber et al., Phys. Lett. B 442 (1998) 443 [arXiv:nucl-th/9808021].

[65] X. Zhu, H. Petersen and M. Bleicher, AIP Conf. Proc. 828 (2006) 17. 